\title{
EL ALUVIÓN COMIENZA A SEDIMENTAR
}

POR

\author{
JESÚS LABRADOR FERNÁNDEZ
}

Los flujos de inmigrantes en España han sido continuos y crecientes en los últimos quince años. Este proceso tiene como posible consecuencia la consolidación de minorías en nuestro país. Este artículo trata de analizar algunos de los procesos de asentamiento y consolidación de una población, la ecuatoriana, que ha protagonizado un flujo significativo de entrada. Se centra fundamentalmente en las estrategias de adaptación en los ámbitos familiares y educativos de los hijos de inmigrantes ecuatorianos.

Palabras Clave: Hijos de inmigrantes, ecuatorianos, minorias, estilos de crianza, educación.

\section{EL ALUVIÓN COMIENZA A SEDIMENTAR}

Estamos en el comienzo de uno de los procesos históricos más llamativos y relevantes que va a tener que afrontar nuestra sociedad, nos referimos al proceso que transforma un país de inmigración en un país de minorías. Un proceso en el que se construirán y por tanto se diversificarán las minorías de una España cada vez más compleja. Entendemos aquí por minoría lo propuesto por Hillman en su Diccionario de Sociología: «grupo o sector de la población de una sociedad o de un Estado que, a causa de sus características sociales, culturales, lingüísticas, religiosas o étnicas se diferencian de la mayoría de la población (...) la permanencia viene determinada por el grado de esfuerzo para mantener y proteger la propia identidad» ${ }^{1}$.

Las minorías, sus relaciones con la mayoría y las relaciones entre las minorías que se están configurando van a ser elementos de la convivencia social

1 Hillman, 2001: 585. 
muy relevantes y determinantes en los próximos años en nuestro país. En este proceso el colectivo de inmigrantes ecuatorianos es uno de los protagonistas más activos y destacados.

La inmigración ecuatoriana a España es un fenómeno relativamente reciente y posee unas características diferenciales tales que hacen de ella un fenómeno demográfico, económico y social relevante y diferenciado. En este artículo vamos a abordar alguna de esas características diferenciales como son la intensidad del flujo hacia nuestro país, la estructura sociodemográfica de la población que se ha asentado y por último en los tipos de proyecto migratorio construidos desde una serie de actitudes que han generado unas estrategias de adaptación bastante exitosas.

\section{EL ALUVIÓN}

La inmigración ecuatoriana hacia España tiene antecedentes registrados desde antes de los años 50, sin embargo lo que hoy conocemos como migración ecuatoriana ha mostrado un flujo especialmente intenso entre los años 1999 hasta 2001.

En este corto período de tiempo la población ecuatoriana en España se visibilizaba de una forma muy repentina y, sorprendentemente debemos decir, esa visibilización tan súbita fue asumida por la sociedad española sin grandes dificultades ni conflictos.

Según Gómez Ciriano el incremento de las cifras que poseemos sobre la población ecuatoriana en España empieza a ser sobresaliente a partir de $1999^{2}$. En ese año se contabilizan 36.684 entradas de ecuatorianos en los puestos fronterizos, desde 1994 las cifras de este mismo índice estaban rondando las diez mil personas. Estas cifras, con ser espectaculares, no recogen en su totalidad la dimensión del fenómeno, ya que en esos años era una estrategia de viaje habitual hacia España el entrar desde otros aeropuertos europeos, como el de Ámsterdam, París o Fráncfort.

En el año 2000 las cifras se multiplican casi por cuatro llegando a las 124.545 entradas de ecuatorianos por la frontera. Son múltiples y complejos los factores que explican este aluvión tan intenso y repentino de población ecuatoriana hacia nuestro país. En estás páginas vamos a apuntar los que consideramos más relevantes, sin olvidar que en todo proyecto migratorio son muchos los factores que determinan la decisión y que ésta la toman personas con sus propias biografías y condicionantes.

\footnotetext{
2 Gómez Ciriano, 2007: 50-82.
} 
En el origen de este súbito flujo es necesario mencionar en primer lugar las terribles crisis económicas y sociales por las que ha pasado el país. Quizás el hito histórico que señala la existencia de una situación social y económica insostenible en el país andino sea la guerra del Cenepa, conflicto fronterizo en la cabecera del río Cenepa, entre Perú y Ecuador, que estalló a comienzos de 1995. Esta breve guerra tuvo consecuencias enormes para la economía ecuatoriana que ya tenía graves problemas, sirva como ejemplo que la inflación de 1994 era de un 27,3\%. El conflicto exigió un esfuerzo económico muy importante que contribuyó a dislocar y a la larga destruir todo el sistema financiero. A esta crisis de origen social hay que añadir el desastre natural del «Niño» que originó grandes pérdidas en el sector primario.

Por último, y no menos importante, en esos mismos años comienza una lamentable crisis de liderazgo político, presidentes como Abdalá Bucaram, Fabián Alarcón, Jamil Mahuad son protagonistas de presidencias impredecibles, que van deteriorando las cifras macroeconómicas hasta llegar a enero de 2000, cuando el presidente Mahuad confirma la hecatombe anunciando la dolarización de la economía ecuatoriana. Lo que ha significado el comienzo de una durísima reconversión de todo el sistema económico financiero del país andino.

Otro elemento que debemos tener en cuenta para explicar este flujo tan explosivo son las condiciones de entrada en nuestro país reguladas por los tratados bilaterales. El marco normativo sumamente favorable para la entrada, para el acceso al mercado de trabajo y para el establecimiento legal en España, hacía de los inmigrantes ecuatorianos una población privilegiada con respecto a otros colectivos. El canje de notas sobre supresión de visados de estancia entre España y Ecuador del año 1963, eximía del paso por los consulados para proveerse del visado, y así anulaba uno de los instrumentos más eficaces de control de flujos. Este tratado no será revisado por los Estados firmantes hasta agosto de 2003. El convenio de doble nacionalidad hispano-ecuatoriano de 1964, que se mantuvo en vigor hasta agosto de 2000, garantizaba el máximo nivel de derechos a aquellos residentes de los países firmantes que por cualquier razón no quisieran acceder a la otra nacionalidad. Esto en la práctica significaba la posibilidad de que todo ecuatoriano, que reuniera los requisitos académicos y profesionales, pudiera trabajar en el mercado laboral español en igualdad de condiciones que los aborígenes. Por supuesto que esto no eximía de la obtención del permiso de trabajo, pero una vez conseguido las condiciones eran muy ventajosas con respecto a otros colectivos de inmigrantes. También existe un convenio de seguridad social entre España y Ecuador desde 1960 y por último los artículos 17 a 18 del Código civil español, que regula el 
acceso con dos años de residencia legal en nuestro país a la nacionalidad de todos aquellos ciudadanos nacidos en un país iberoamericano.

Estas ventajas ayudan a que, a partir de 1994, las solicitudes de trabajo en los contingentes por parte de población ecuatoriana no hacen más que crecer, hasta llegar a los datos de 2000 mencionados más arriba.

\section{Gráfico 1. Porcentaje de variación de Residentes ecuatorianos 3 .}

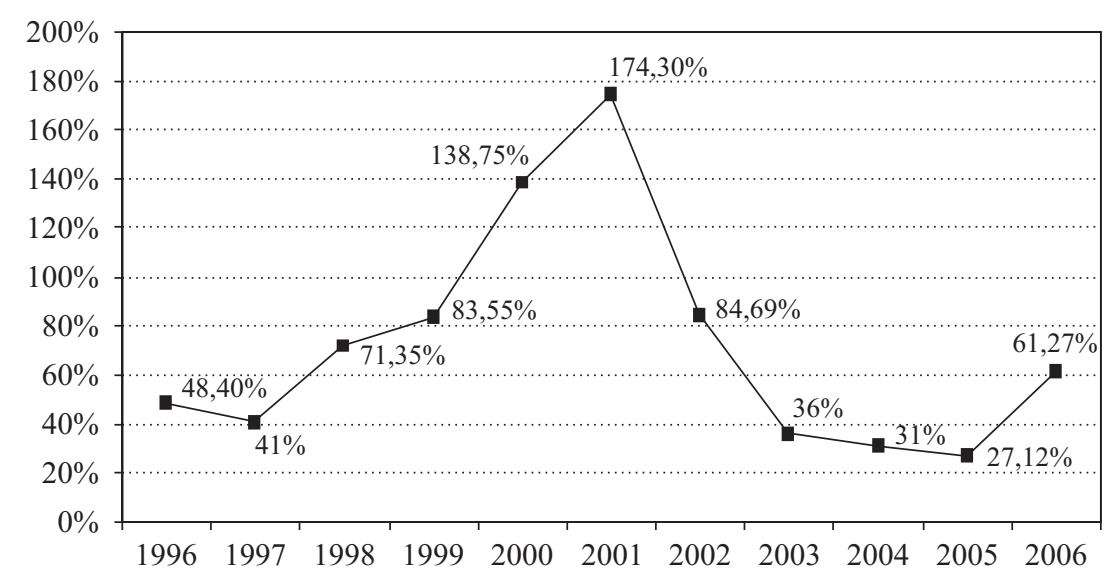

Fuente: Elaboración propia.

Como vemos en el gráfico 1, en 1999 el crecimiento de la población ecuatoriana empieza a ser espectacular. En los datos proporcionados por el Ministerio del Interior se recogen unos aumentos poblacionales superiores al $80 \%$. Todavía más espectacular son los de 2000 y 2001, aunque en este último debemos tener en cuenta la distorsión que supone el proceso extraordinario de regulación llevado a cabo en ese año. Si atendemos a los incrementos sobre la base del Padrón Municipal de Habitantes los datos son todavía más espectaculares ya que, en el año 2000 , el incremento anual fue de $578,8 \%{ }^{4}$.

En cualquier caso, en ese trienio se pasa de un colectivo con una representación poco significativa, no llegaba a las 15.000 personas, a ser ya una población significativa y muy numerosa con respecto a otros colectivos inmigrantes. Quizá el datos que más ilustre esa intensidad tan inusual y característica de

3 Ministerio de Trabajo e Inmigración, 2006.

4 Colectivo IOÉ, 2007: 189. 
este flujo migratorio sea el mencionado más arriba del registro de entrada por lo aeropuertos españoles o el del padrón municipal del año 2000.

A partir de ese año el flujo de entradas sigue siendo intenso y, aunque en agosto de 2003 se empieza a exigir visado, se va creando una importantísima bolsa de personas en situación irregular que tendrán que esperar al proceso de normalización de 2005, para regularizar su situación.

Gráfico 2. Residentes ecuatorianos en España ${ }^{5}$.

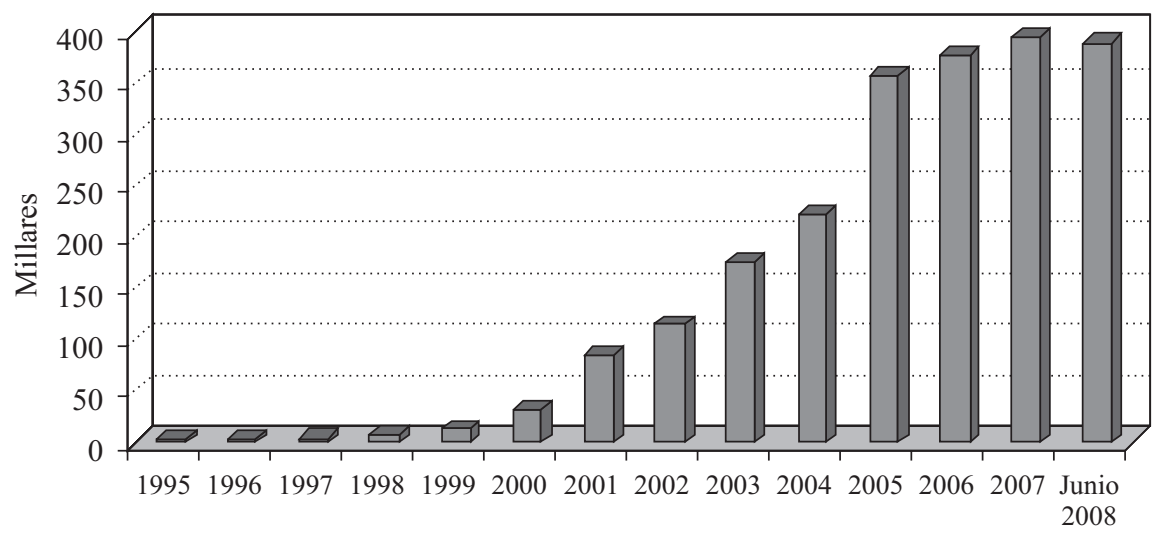

Fuente: Elaboración propia.

Estas entradas, junto a las que han sido originadas por el proceso de reagrupación familiar, nos lleva a que hoy este colectivo representa el tercero en importancia numérica de los que están asentados en nuestro país, sólo por detrás de marroquíes y rumanos. Hay que señalar que la inmigración procedente de Rumanía se ha significado también por un flujo muy intenso en los últimos años, favorecido además por la entrada de este país en la Unión Europea lo que hace que sus nacionales puedan disfrutar de un régimen de residencia comunitario.

Podemos decir que el último proceso de normalización de 2005 ha significado para la población ecuatoriana en España la culminación de un período de estabilización demográfica en nuestro país. Las bolsas de ecuatorianos en situación irregular fueron drásticamente reducidas gracias a este proceso que, junto a una situación en el país de origen ya no tan crítica, han hecho que el flujo en estos momentos recupere índices de crecimiento moderados y

\footnotetext{
5 Ministerio de Trabajo e Inmigración, 2008.
} 


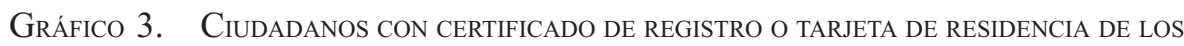
PAÍSES DE ORIGEN MÁS SIGNIFICATIVOS EN LA INMIGRACIÓN EN ESPAÑA ${ }^{6}$.

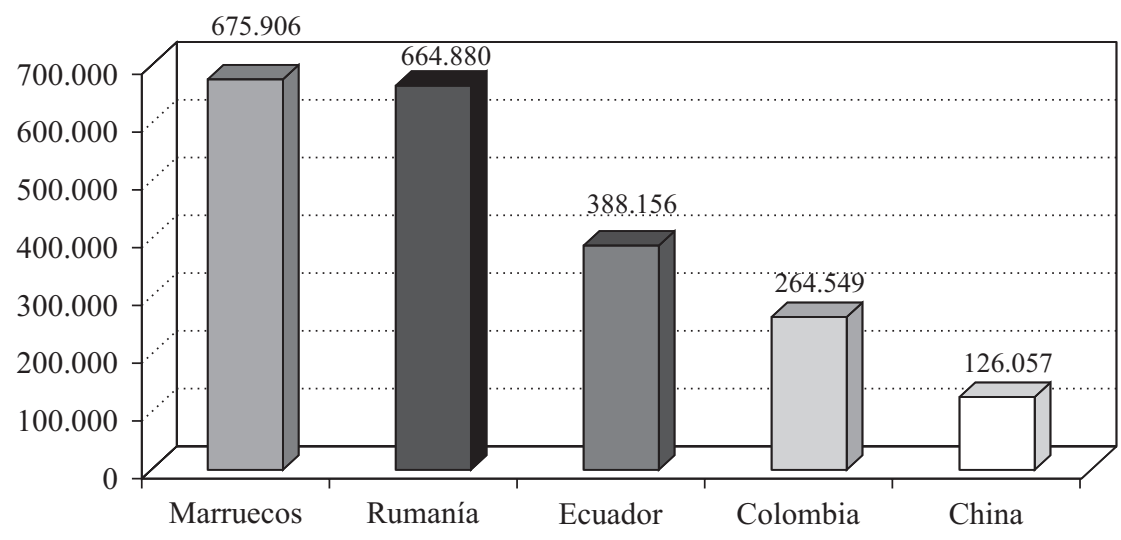

Fuente: Elaboración propia.

los procesos de asentamiento y estabilización en España se hayan puesto en marcha.

Hay que hacer notar que, según los datos de residentes con permiso del Ministerio de Trabajo e Inmigración de junio de 2008, por primera vez en la historia de la migración reciente ecuatoriana, el número de residentes ha descendido. Posiblemente éste es uno de los primeros efectos de la crisis económica por la que está pasando nuestro país y debemos esperar para saber, a ciencia cierta, hasta dónde llegan esas consecuencias en la población ecuatoriana.

\section{ESTRUCTURA DEMOGRÁFICA}

En cuanto a las características demográficas de esta población podemos decir que es una de las más equilibradas en cuanto a sexos, aunque en los primeros años del aluvión se recogen datos que apuntan a un desequilibrio en favor de las mujeres, es necesario tener en cuenta que también en esos momentos las bolsas de población que carecía de papeles y que por tanto no estaba registrada era muy grande, y que las oportunidades de trabajo estable estaban más centradas en el servicio doméstico, con lo que, podemos suponer, que el

\footnotetext{
${ }^{6}$ Idem.
} 
desequilibrio mencionado, fue motivado por un más fácil acceso a la regularización de las mujeres que de los hombres.

Es una población que comenzó siendo muy joven y que con el paso de los años, como no podía ser de otra manera, se ha ido transformando en una población madura con una natalidad que está logrando que los menores de 15 años estén creciendo significativamente.

Gráfico 4. PirÁmide de población Residentes eCUatorianos en España. MARZO de 2008 .

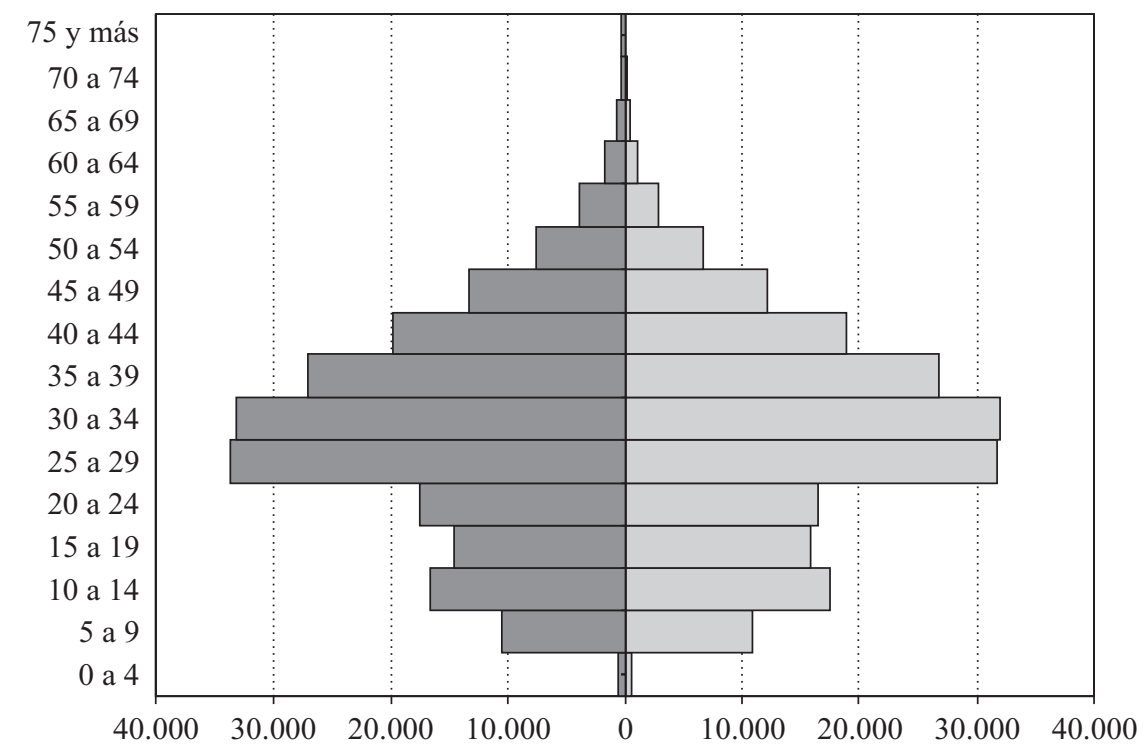

Fuente: Elaboración propia.

\section{UN PROYECTO MIGRATORIA FAMILIAR ESTABLE Y A LARGO PLAZO}

Estamos ante una migración en la que la familia se constituye como el eje sobre el que se articula su estrategia migratoria. Estrategia que suponemos, por los indicadores que expondremos a continuación, es de estabilidad a largo plazo. Posiblemente la crisis económica por la que estamos comenzando a discurrir en los países occidentales sea la piedra de toque que confirme o desmienta esta hipótesis. Los inmigrantes ecuatorianos se plantean una proyecto migrato-

\footnotetext{
${ }^{7}$ Idem.
} 
rio en el que se apuesta por que los sacrificios realizados al salir del país y acometer la aventura migratoria van a ser recompensados no en ellos mismos, sino en sus descendientes. Estamos ante una población con una clara estrategia de permanencia, en la que se asumen sacrificios y sufrimientos no con el fin de un éxito social inmediato, sino con la idea de mejorar el estatus y el futuro de los hijos. Como mostraba Fresneda los ecuatorianos se presentaban a sí mismos como aquellos que venían a sufrir y a diferencia de otros colectivos latinoamericanos, los ecuatorianos no percibían un descenso en su estatus ni manifestaban sorpresas respecto al trato dado por los españoles ${ }^{8}$. El sufrir, el aguantar era algo con lo que contaban y que adquiría sentido en las oportunidades de movilidad social ascendente que da España no a ellos mismos, sino a su descendencia. El éxito de este objetivo pasa por la estabilidad de su peripecia migratoria.

Según datos del Colectivo Ioé, otro de los indicadores que apunta en la misma dirección de la búsqueda de cierta estabilidad y permanencia son las cifras sobre los matrimonios contraídos ${ }^{9}$. Entre 1996 y 2005 contrajeron matrimonio 16.325 ecuatorianos. Además hay que tener en cuenta que existe un porcentaje significativo de matrimonios mixtos, sobre todo de mujeres ecuatorianas con hombres españoles (en 2005 un $48 \%$ de los esposos de ecuatorianas fueron españoles).

En este proceso de estabilización es también significativa la forma en que se han ido distribuyendo por el territorio nacional. La población ecuatoriana ha experimentado una notoria fuerza centrífuga. En los primeros años se produjo un asentamiento muy concentrado en Madrid, y en pequeñas ciudades agrarias del sureste español, en las que los empresarios de la agricultura intensiva comenzaron a sustituir la mano de obra marroquí por la ecuatoriana, con el pretexto de la «proximidad cultural». En Madrid se concentraba el 40\% de los ecuatorianos con permiso de residencia en 2003, donde encontrábamos un perfil mayoritario trabajando en el servicio doméstico (mujeres en su mayoría) y la construcción (varones). De esta situación de clara concentración, se ha pasado a una proporción menos relevante de los residentes en la capital. En 2008 los ecuatorianos residentes en Madrid suponen un 27\%, viven en Cataluña un $20 \%$, un $12 \%$ en Murcia, un $6 \%$ en Andalucía y un 3\% en Navarra.

Otro elemento que empieza a ser significativo en el colectivo ecuatoriano, y que viene a reforzar la idea de un asentamiento estable, es el dato aportado por el Informe Global Entrepreneurship Monitor ${ }^{10}$ donde se señala que los in-

\footnotetext{
8 Fresneda, 2002.

9 Colectivo IOÉ, 2007: 195-197.

10 De la Vega, 2008: 103.
} 
migrantes procedentes de Ecuador ocupan el primer puesto, en nuestro país, como emprendedores empresariales. Ecuador, según los datos del Informe, es el país que ha aportado mayor proporción de emprendedores extranjeros en España, 12,8\% en el 2007, desplazando a Argentina, que había ocupado el primer lugar en 2005 y 2006.

Quizá el dato más significativo y simbólico de esta estrategia de asentamiento estable y a largo plazo adoptada son las cifras sobre los procesos de nacionalización. Vemos que el incremento de sujetos ecuatorianos que obtienen la nacionalidad española entre los años 2005 y 2006 es del 94,17\%, siendo el incremento para todos los originarios de Iberoamérica, en ese mismo período, un $60 \%$. La tasa de incremento para todos los ciudadanos de origen extranjero que se nacionalizaron en ese período fue de un 45\%. En el año 2006 han obtenido la nacionalidad española 19.477 ciudadanos que tenían previamente la ecuatoriana. Éste es el valor absoluto mayor de todas las nacionalizaciones resueltas en nuestro país y supone un $32 \%$ del total de nacionalidades concedidas durante ese año. El siguiente país de procedencia con más ciudadanos nacionalizados es Colombia con 12.720 en ese mismo período ${ }^{11}$.

$\mathrm{Si}$ atendemos a los datos de nacionalización por edades vemos que siempre la población ecuatoriana tiene porcentajes superiores de personas nacionalizadas en todos los tramos de edad inferiores a 34 años. Estamos ante una población joven, con un largo recorrido para construir su vida y optan por construirla con la nacionalidad española.

\section{¿LA NUEVA MINORÍA?}

Como venimos señalando a lo largo de estas páginas, el proyecto migratorio generalizado de los ecuatorianos es fundamentalmente intergeneracional. Son los menores, hijos de los que han tomado la decisión, los beneficiarios principales de todo este proyecto. Desde esta perspectiva nos parece muy relevante el comenzar aproximarse a estos jóvenes depositarios de toda esa «misión» que han de cumplir.

En el Instituto Universitario de Estudios sobre Migraciones llevamos trabajando en los últimos años sobre las trayectorias de incorporación a la vida adulta de los jóvenes hijos de inmigrantes, los jóvenes hijos de ecuatorianos son unos de los protagonistas de esos trabajos ${ }^{12}$, y en ellos hemos encontrado

11 Ministerio de Trabajo e Inmigración, 2006.

12 Trayectorias educativas y de inserción laboral de jóvenes inmigrantes. Proceso de transición a la vida adulta. Estos estudios han sido posibles por la cofinanciación del Ministerio de 
una serie de características diferenciales que son las que van a ocupar las siguientes líneas.

Según nuestros datos ${ }^{13}$, casi el $50 \%$ de los jóvenes ecuatorianos que se encuentran en nuestro país llegaron entre los 13 y los 17 años, éste es un dato especialmente significativo ya que ésta es, posiblemente, la peor edad para acometer un cambio tan radical de referentes y de vinculaciones sociales. Hay que tener en cuenta que estamos ante adolescentes que no han tomado la decisión, con lo que, en muchos casos, son traídos contra su voluntad, lo que manifiestan en las entrevistas es que ellos estaban bien en Ecuador y que el venir a España supuso, cuando menos, una contrariedad.

Es muy frecuente que existan jóvenes que han permanecido separados de sus padres quedando al cuidado de otros familiares, en estos casos, la migración y posterior adaptación es todavía más compleja. Si la separación ha sido larga (en nuestra muestra más de un $40 \%$ de los jóvenes encuestados manifiestan haber estado separados de sus padres más de tres años), ya se han producido los reajustes y adaptaciones para vivir es esa situación. Situación que significa una ruptura en las relaciones familiares, pero que tiene asimismo algunas ventajas. No han sido pocos los jóvenes que nos han narrado como vivían mucho mejor en su país, sin una autoridad parental próxima y además con los regalos que les hacían llegar desde Europa, con la consiguiente diferenciación entre su grupo de iguales.

Mencionamos todos estos elementos para mostrar que los procesos migratorios cuando se realizan en edades adolescentes y preadolescentes son más complejos, dificultosos y conflictivos. Es necesario tener en cuenta que al menor, por su corta edad, le caracteriza su escasa dotación de experiencia, una estructura de vinculaciones en la que son todavía muy dependientes, y unos procesos cognitivos que están comenzando a alcanzar la madurez. A todos esos elementos hay que añadir en los jóvenes hijos de inmigrantes que están siendo sometidos a un cambio radical en su vida, su capacidad de respuesta a un entorno tan exigente es cuando menos limitada.

En relación con los procesos de reagrupación familiar en la adolescencia, todos los jóvenes reagrupados manifiestan en mayor o menor grado, que la llegada a España en las condiciones mencionadas les produjo un desajuste fuerte

Trabajo y Asuntos Sociales — actualmente Ministerio de Trabajo e Inmigración- y el Fondo Social Europeo, dentro del programa Régimen General de Subvenciones, convocatorias 2004, 2005 y 2006. La investigación ha sido realizada por el autor y María Rosa Blanco.

${ }^{13}$ La muestra estaba formada por 695 jóvenes nacidos en Marruecos 175, China 93, República Dominicana 86, Ecuador 121 y España 220, con edades comprendidas entre los 16 y los 24 años. 
en tres dimensiones diferentes: la personal, la familiar y la escolar. A nivel personal supone una pérdida de su rutina en el país de origen: abuelos y tíos se encargaban de ellos y la vida transcurría sin mayores dificultades, más aún, vivían en mejores condiciones económicas gracias a las remesas de sus progenitores. A nivel familiar la reagrupación les enfrenta con unos progenitores que ya no conocen, que recordaban de pequeños y de los pocos viajes que hacían y que han cambiado en los años que llevaban aquí. En la escuela se enfrentan de un día para otro con un ámbito totalmente desconocido, por lo general mucho más exigente en cuanto a lo académico y mucho más laxo y permisivo en cuanto a la disciplina y convivencia.

Teniendo en cuenta estas premisas los investigadores esperábamos encontrar claros y contundentes índices de riesgo social en estos menores. Los jóvenes ecuatorianos están en estos momentos acometiendo un complejo y difícil proceso de adaptación en el que se encuentran en una posición confusa que parte de esas condiciones mencionadas más arriba y que les lleva a tener que vivir en un mundo distinto al de sus raíces, pero en el que deben tener éxito para dar sentido al proyecto migratorio de sus padres. Estamos ante un proceso largo y dinámico que se está produciendo en estos momentos y que, como decíamos al comienzo, es el que generará un Estado con ciudadanos de pleno derecho y que, a la vez, podrán pertenecer a una minoría saliente, definitoria y discriminable. Hoy por hoy no podemos saber si la inmigración ecuatoriana formará o no formará parte de una de esas minorías. Abordaremos seguidamente algunos fenómenos característicos de la socialización de los menores ecuatorianos. Nuestra intención no es hacer una predicción de la posible creación de minorías, para ello deberíamos contemplar muchos mas datos, no sólo de los ecuatorianos sino también de la mayoría y la interacción que generan. Nuestra intención es ir desvelando algunos riesgos y oportunidades que podemos comenzar a percibir.

Los ejes fundamentales sobre los que se articula la socialización y el crecimiento de estos menores son: la familia y la escuela. En función de cómo hayan transcurrido los sucesos biográficos dentro de esos dos ámbitos, así estos jóvenes diseñarán y organizarán sus trayectorias hacia la vida adulta.

Ya hemos señalado la importancia de la estructura familiar en las trayectorias migratorias de este colectivo, como no podía ser de otra forma la familia es esencial para explicar los procesos de adaptación de los jóvenes hijos de inmigrantes. En nuestros trabajos las familias ecuatorianas se han revelado con una serie de características diferenciales con respecto a otros colectivos de inmigrantes. La familia es para la mayoría de los seres humanos el primer contexto en el que acontece su existencia, contexto de dramática relevancia, dadas las características ontogenéticas de nuestra especie. Por otra parte, es el grupo 
primario en el que se aprende a ser. Una de las principales y más críticas funciones de las familias es la socialización de los hijos. Es el principal agente mediador y, por tanto, escultor del desarrollo del sistema afectivo y cognitivo de los nuevos miembros de la especie.

En el trabajo de investigación que inspira este artículo hemos explorado el juicio que hacen los menores de esa función socializadora de sus padres ${ }^{14}$. Hemos de decir que el colectivo de menores ecuatorianos es el que más nos ha sorprendido, por lo positiva y elevada opinión que tienen respecto a sus padres. Tanto padres como madres son vistos por sus hijos con puntuaciones muy elevadas en su capacidad de dar afecto, esta evaluación, hemos de decir es muy similar a la que hace el resto de los colectivos, y como todos ellos plantean puntuaciones mayores para las madres que para los padres. Las diferencias comienzan a surgir cuando pedimos a los jóvenes que evaluaran la capacidad de hacer críticas y reprochar las conductas de los hijos. Los jóvenes ecuatorianos puntúan por encima de sus compañeros de muestra. Los padres ecuatorianos son vistos por sus hijos con los valores más altos en esta capacidad de manifestar las críticas, las censuras y las reprobaciones, las madres, aunque son vistas como menos críticas que los padres, obtienen también puntuaciones más altas que las del resto de los colectivos nacionales estudiados.

Vemos, por tanto, cómo los jóvenes ecuatorianos nos describen un estilo de manejo de afectos en sus hogares donde los padres son capaces de dar y expresar afecto, calidez y amor, pero también son capaces de hacer la misma expresión de la crítica y la censura. Estamos sólo ante una simple puntuación en una escala pero es posible que esta puntuación, que difiere de las de los otros colectivos, nos esté marcando un estilo peculiar de manejo de los afectos en los hogares ecuatorianos en los que existe una alta implicación emocional sin dejar de lado las labores de supervisión. La supervisión y el control de las conductas de los hijos son tareas esenciales en todo el proceso de socialización.

Hay que tener en cuanta que cuando hablamos de población inmigrante, en general son comunes unas condiciones sociolaborales en las que las jornadas laborales de los progenitores suelen ser tan extensas, que las relaciones pater-

14 En nuestros trabajos con jóvenes hijos de inmigrantes hemos utilizado dos escalas sobre relaciones paternofiliales. Nuestra intención era aproximarnos a los estilos de socialización que existen entre las familias inmigrantes. En primer lugar utilizamos la escala de afecto (EA) (Fuentes, Motrico y Bersabé, 1999) que se compone de dos factores: afecto-comunicación y crítica-rechazo. Utilizamos asimismo la escala de normas y exigencias (ENE) (Fuentes, Motrico y Bersabé, 1999). Esta escala tiente tres factores: forma inductiva, forma rígida y forma indulgente. Se refieren naturalmente a las formas que tienen padre y madre de establecer las normas y de exigir su cumplimiento. 
no filiales se ven drásticamente disminuidas, con lo que los procesos de supervisión se encuentran amenazados. Pero esta ausencia física no suele estar sola, es habitual que esté acompañada por una falta de disponibilidad psicológica motivada por las altos índices de estrés sociolaboral, ansiedad y depresión de los padres. Las herramientas utilizadas para la obtención de la información nos proporcionaban la posibilidad de distinguir tres tipos básicos de supervisión: inductiva, rígida y negligente. La forma inductiva es aquella en la que las normas y reglas que deben cumplir los hijos son explicadas, razonadas y cargadas de sentido por la autoridad parental, que siempre esta dispuesta al diálogo y el razonamiento. La forma rígida es en la que impera una autoridad inflexible, con muchas normas y muy presta a castigar ante la mínima infracción. La forma negligente, posiblemente la que peores consecuencias genera en el desarrollo de los niños, es aquella autoridad errante, confusa y muy pasiva.

Pues bien, nuestros jóvenes ecuatorianos ven a sus padres fundamentalmente con un estilo inductivo tanto para los padres como para las madres, aunque son ellas las que aparecen como más razonadoras. Hay que hacer notar que las puntuaciones en este estilo de supervisión están por encima del resto de los colectivos inmigrantes estudiados. Curiosamente, aunque con puntuaciones inferiores, también son los padres y madres ecuatorianas las vistas como más rígidas y autoritarias con respecto al resto de padres. En cuanto al estilo indulgente el es menos utilizado según la opinión de sus hijos, y lo son también en puntuaciones inferiores a los otros padres analizados.Los padres ecuatorianos son vistos por sus hijos como los agentes que tienen más importancia en la orientación de sus estudios, en el resto de los colectivos analizados esta relevancia de los padres como referentes útiles para tomar decisiones es menor. Todos estos datos nos conducen a la idea de que una supervisión activa es característica de las familias ecuatorianas, además hay que tener en cuenta que una supervisión eficaz es aquella que es percibida y tenida en cuenta por los supervisados.

Es necesario hacer estudios más profundos y sutiles sobre los estilos de socialización de las familias inmigrantes, pero en esta breve aproximación se están planteando unas tendencias diferenciales que nos apuntan a que, aún con todos los condicionamientos sociales y económicos que amenazan las tareas familiares, aún con todos los difíciles procesos de adaptación y readaptación que supone la migración y la reagrupación familiar, estamos ante familias que son capaces de dar tanto afecto, cariño y cercanía, como criticar, reprender y supervisar de forma activa la conducta de sus hijos. Hijos que, volvemos a decir, son los supuestos y últimos beneficiarios de toda la peripecia migratoria. Parece que estamos ante familias que acometen su función socializadora de una forma activa y enérgica. 
Existe una circunstancia contextual que enmarca todas estas prácticas socializadoras y que es imposible eludir, nos referimos a las actitudes y creencias generales que los miembros de la sociedad de origen tienen con respecto a la inmigración. Esta «atmósfera de recepción» se extiende poco a poco hacia los niños y afectan sus percepciones, identidades en desarrollo y conductas. Son los padres en muchos casos los que ejercen un papel mediador entre este clima y sus hijos, de esta forma los menores van construyendo el significado del nuevo medio social en el que les está tocando vivir.

La atmósfera, en general, está muy contaminada por un sinnúmero de estereotipos y miedos que hacen que las estrategias de segregación suelan ser en muchas ocasiones las más adaptativas. Si las relaciones entre aborígenes e inmigrantes están presididas por la desconfianza y la segregación, el discurso que organiza la cosmovisión del menor también estará impregnado de desconfianza y segregación. Los padres que han tenido una buena historia de relaciones con los aborígenes proporcionan a sus hijos una protección enorme contra los reflejos sociales negativos; sin embargo, hay familias, con historias muy negativas y desafortunadas, en las que esas imágenes tóxicas son reforzadas y ampliadas con lo que el clima de crianza es un continuo proceso de reforzamiento de estrategias defensivas y marginadoras.

La escuela es al otro gran ámbito de socialización por los que pasan los menores. Está de más señalar la importancia de los procesos educativos, instructivos y formativos que se producen a través de la escolarización, máxime cuando nos referimos a una población infantil y juvenil, producto de la inmigración, cuyo primer contacto sistemático con la nueva cultura se produce en la escuela. Tal y como señalan los Suárez-Orozco, el proceso de adaptación a la escuela y los resultados obtenidos constituyen un predictor significativo del bienestar futuro del joven y de las aportaciones que haga a la sociedad ${ }^{15}$.

Lo primero que se debe decir en cuanto a la educación reglada es que no hay acceso a los datos escolares, ya sea éxito o fracaso, desglosado por el origen nacional de los alumnos. No podemos hacer comparaciones atendiendo a las diferencias nacionales en las estrategias de adaptación a la escuela que pudieren ser antecedentes de diferencias en resultados académicos. Nuestro sistema educativo está diseñado para dotar a la población en él inserta de una estructura de oportunidades igualitaria... sabemos que esa pretensión no es más que eso, una pretensión. El estatus sociocultural de los colegios de nuestro país es muy distinto y los resultados que en ellos se producen también lo son. La entrada de población inmigrante al sistema educativo español no hace más

15 Suárez-Orozco, 2003. 
que acentuar la diversidad llegando a la polarización. Por un lado encontramos colegios de estatus elevado a los que no tienen acceso alumnos que puedan significar problemas o complicaciones, y por el otro existe una mayoría de colegios que se están viendo desbordados por una afluencia de complejidad para la que nadie está preparado. No descubrimos nada nuevo si decimos que en este ámbito se están dando fenómenos de segregación frente a la diversidad.

El sistema educativo español está configurado en dos grandes períodos: la enseñanza obligatoria y la no obligatoria. La obligatoria comprende la Educación Primaria de 6 a 12 años y la Educación Secundaria Obligatoria, ESO, de 12 a 16. La educación infantil aunque muy extendida y universal, sobre todo en el segundo ciclo de 3 a 6 años, no es obligatoria y presenta en nuestro país una fuerte demanda que no logra satisfacer totalmente el sistema público. A partir de los 16 años comienza la secundaria no obligatoria que, fundamentalmente, está compuesta por el Bachillerato y por los Ciclos Formativos de Formación Profesional. Una vez terminados estos estudios se puede acceder a la Universidad. Según datos del Ministerio de Educación Política Social y Deporte del curso 2005-2006 Ecuador era el país que aportaba una relación porcentual más elevada con respecto al total de la población extranjera, en cuanto a alumnos matriculados en educación no universitaria.

Gráfico 5. Evolución de AlumNos Matriculados EN ENSEÑANZAS NO UNIVERSITARIAS ${ }^{16}$.

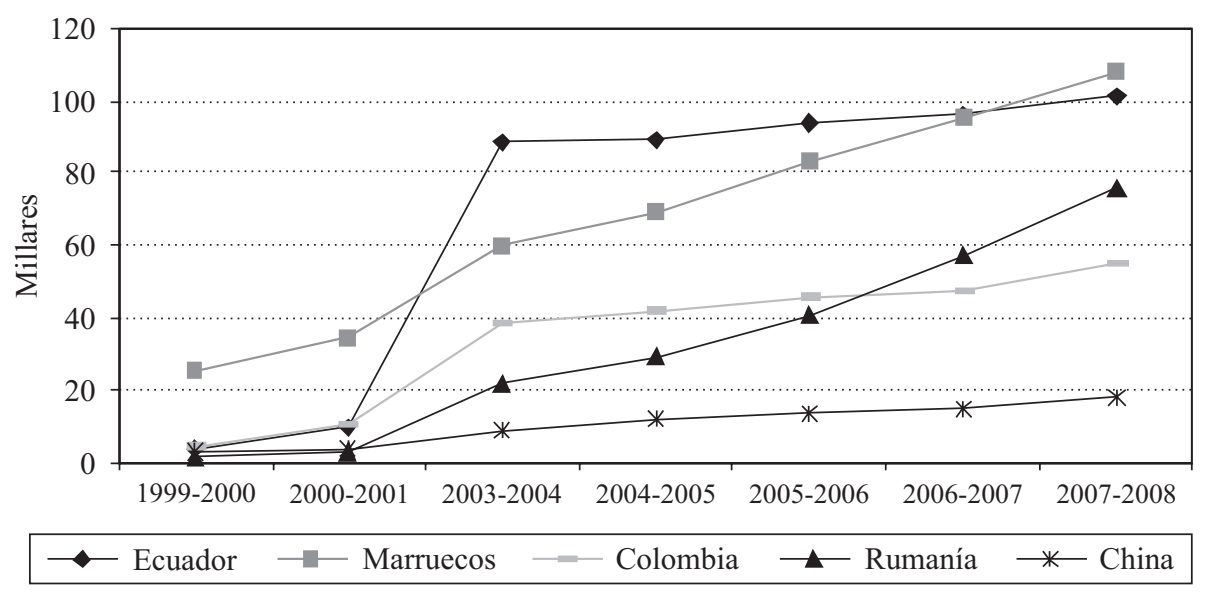

Fuente: Elaboración propia.

16 Ministerio de Educación, Política Social y Deporte, 2008. 
El alumnado ecuatoriano está matriculado mayoritariamente de los niveles altos de la enseñanza obligatoria, el 30\% de los alumnos matriculados en enseñanzas no universitarias lo está en la Enseñanza Secundaria Obligatoria en el curso 2007-2008, en números absolutos representaban el colectivo nacional más numeroso en la ESO con 29.449 menores $^{17}$. Debemos recordar que un gran porcentaje de menores ecuatorianos han hecho el viaje en edades cercanas a la adolescencia, con lo que se han debido incorporar al sistema educativo en los últimos años de primaria o en la secundaria. Por tanto, es la enseñanza secundaria la que más nos interesa si queremos hacernos una idea del estado de la escolarización de esta comunidad. Sin embargo el elemento más significativo del tipo de proyecto educativo es la enseñanza secundaria no obligatoria. Continuar estudios en edades en las que es posible la incorporación al mercado laboral es una opción muy minoritaria entre los jóvenes inmigrantes, e implica una renuncia a unos ingresos inmediatos por el valor intangible de obtener una mejor inserción laboral unos años después.

La opción mayoritaria confirmada por los datos es la de incorporarse al mercado laboral, si en la ESO, en el curso 2006-2007, había matriculados 29.449 alumnos ecuatorianos, en las enseñanzas secundarias no obligatorias el número se había reducido a 7.661. Pero debemos ver este dato en su contexto y en comparación con el resto de los colectivos inmigrantes. Los jóvenes ecuatorianos presentan tasas de matriculación superior al resto de los jóvenes inmigrantes en las enseñanzas secundarias no obligatorias. El 19\% de los jóvenes ecuatorianos encuestados en nuestro trabajo han superado los estudios secundarios no obligatorios, sólo la población china tiene índices mayores. Hay que tener en cuenta que estamos ante un colectivo con poco tiempo en nuestro país y que una de las tendencias más relevantes en nuestros trabajos es la alta correlación entre el tiempo en España y el nivel de estudios alcanzados.

Si acudimos a los datos proporcionados por los ministerios competentes podemos apoyar esta idea de una mayor continuidad en los estudios. Hay que tener en cuenta que los rangos de edad que podemos comparar para averiguar tasas de matriculación no coinciden con las edades de entrada y de salida en la secundaria no obligatoria, así el Ministerio de Trabajo e Inmigración utiliza grupos de edades quinquenales con lo que el grupo que más se aproxima a las enseñanzas secundarias no obligatorias sería el de 15 a 19 años, cuando éstas comprenden desde los 16 a los 18 en la opción del bachiller y puede llegar a los 20 en los ciclos superiores de la formación profesional, cursos con los que se trabaja en los datos del Ministerio de Educación. Teniendo en cuenta este

\section{Idem.}


desfase de edades y cursos podemos estimar que un $25 \%$ de los jóvenes ecuatorianos en edad de hacer enseñanzas secundarias no obligatorias está matriculado en ellas. Éste es el porcentaje más alto en comparación con el resto de colectivos con una población numerosa en nuestro país. La estimación para los marroquíes es de un $12 \%$ y no llega al $10 \%$ para rumanos y chinos. Los colombianos son los únicos que se le acercan con un 23\%. Esta misma estimación de tasa de matriculación para los jóvenes españoles es de un $49 \%$.

En el sistema educativo español existe un sistema alternativo para favorecer la inserción laboral de aquellos jóvenes que muestran fracaso continuado en la ESO, nos referimos a la llamada Garantía Social o Programas de Inserción Profesional. Estos programas son además un dispositivo para aquellos que se incorporan por primera vez al sistema educativo con 16 años. En estos programas la población ecuatoriana es significativa, en el curso 2006-2007 estaban matriculados 1.068 alumnos, pero si lo comparamos con el resto de los colectivos señalados el porcentaje de alumnos en estos programas es de un $1 \%$ igual o inferior al resto de los colectivos analizados. Todos estos datos nos hacen pensar que estamos ante una estrategia educativa con una mayor motivación de continuidad y logro. Posiblemente los efectos de la socialización familiar se están dejando notar en las trayectorias educativas.

Es muy osado predecir lo que va a ocurrir en los próximos años, y menos en una sociedad tan dinámica e interconectada como la actual, pero parece que la población «hispanoecuatoriana» que está comenzando a formarse lo está haciendo desde la clara vocación de movilidad social ascendente con una buena capacidad de sacrificio y de tolerancia para mantener los esfuerzos. Estás características son muy apreciadas y muy adaptativas en los sistemas meritocráticos modernos, con lo que podríamos suponer que esa movilidad social ascendente es posible.

Por otra parte, podemos apreciar el peligro de que en la estructura laboral se puedan producir fenómenos de exclusión o segregación, si hay techos que impiden la movilidad laboral y el acceso a oportunidades económicas, la perdida de significado del esfuerzo en la educación será sensible. Hay evidencias empíricas y sociales de otros países con mayor experiencia migratoria ${ }^{18}$ en las que se constata, desgraciadamente, que la misma cualificación no conduce a las mismas oportunidades para la población joven inmigrante. Esta desigualdad puede tener como efecto secundario un descenso en la motivación y en los resultados educativos de las generaciones que se encuentran en formación. Si

18 López, 5/1 (Londres, 2002): 67-89. Fuligni y Hardway, 14/2 (Princeton, 2004): 99-121. Truong, 2004. 
su expectativa se ve truncada su nivel de frustración se eleva y el del esfuerzo disminuye.

Esta situación de desigualdad asumida de una forma resignada por los inmigrantes de primera generación, es mucho más humillante y generadora de conflictos en personas educadas en nuestro país, en sus valores de igualdad y universalidad y con expectativas de logro (consumo) muy elevadas. Los inmigrantes que se están asentando no sólo nos aportan su trabajo, sobre el cual se ha apoyado buena parte de nuestra incipiente riqueza, sino que también están aportando y creando un nuevo país con minorías que en muy poco tiempo serán minorías de ciudadanos españoles de pleno derecho. La forma en que los jóvenes se incorporan a la vida adulta es esencial en la configuración de la vida social de los próximos años.

Hace años, todos las publicaciones que abordaban los movimientos migratorios en España, incluían alguna referencia a la brusca transición que había sufrido nuestro país de ser un país de emigración a uno de inmigración. Esa transición ya está en nuestra historia y es bueno empezar a tener en cuenta que ahora la transición que nos concierne es la de un país de inmigración a otro de minorías. No podemos decir que este país no haya tenido hasta ahora experiencia en articular la convivencia entre distintas minorías, los gitanos serían los primeros que se nos vienen a la cabeza, pero diversidad étnica ha existido siempre y con diferentes grupos, aunque hayan sido muy minoritarios (maragatos en el oeste y chuetas en el este serían dos ejemplos de esa diversidad). Tampoco podemos decir que toda esa experiencia haya proporcionado a la sociedad española unas competencias ajustadas y eficaces para afrontar los retos de esa convivencia. Los dados están en el aire y el futuro de nuestra convivencia social es lo que está en juego. Un futuro que comienza mañana y que está presidido por una radical transformación social. Estábamos acostumbrados a la convivencia en la homogeneidad, o al menos en cierto espejismo de homogeneidad. Esta circunstancia ya es del pasado. Españoles con fenotipos diversos, con religiones distintas y con tradiciones culturales muy diversas configurarán la ciudadanía de nuestro país.

\section{BIBLIOGRAFÍA}

Borjas, George J., «The Intergenerational Mobility of Immigrants», Journal of Labour Economics, 11 (Chicago, 1993): 113-135.

Cachón, Lorenzo, «Desafíos de la Juventud inmigrante en la nueva España inmigrante», Estudios de Juventud, 60 (Madrid, 2003): 9-32. 
Cachón, Lorenzo, Inmigrantes jóvenes en España. Sistema educativo y mercado de trabajo, Madrid, Injuve, 2003.

Camarero, L. A. y García Borrego, Iñaki, «Los paisajes familiares de la inmigración». Revista Española de Sociología, 4 (Madrid, 2004): 73-198.

Colectivo IOÉ, «La inmigración ecuatoriana en España: una visión a través de las fuentes estadísticas», Emiliano Gomez-Cirano, Andrés Tornos y Colectivo IOÉ, Ecuatorianos en España. Una aproximación sociológica, Madrid, Observatorio Permanente de la Inmigración, 2007: 181-255.

De la Vega, Ignacio, «Global Enterpreneuship Monitor». Informe ejecutivo GEM España 2007, Online . 14-10-2008 <http://www.ie.edu/GEM/img/ informes/34.pdf>

Fresneda, Javier, Ecuatorianos en España: la construcción comunitaria de relatos saludables, Tesis Doctoral, Madrid, Universidad Pontificia Comillas, 2002.

Fuentes, María Jesús, Emma Métrico y Rosa Bersabé, Escala de Afecto (EA) y Escala de Normas y Exigencias (ENE): Versión hijos y versión padres, Málaga, Universidad de Málaga, 1999.

Fuentes, María Jesús, Emma Métrico y Rosa Bersabé, «Análisis psicométrico de dos escalas para evaluar estilos educativos parentales», Psicothema, 13/4 (Oviedo, 2001): 678-684.

Fuligni, Andrew. J. y Hardway, Christina, «Preparing diverse adolescents to become productive adults», The Future of Children, 14/2 (Princeton, 2004): 99-121.

Hillmann, Kart-Heinz, Diccionario enciclopédico de sociología. Barcelona, Herder, 2001.

Gómez Ciriano, Emilio, «Ecuatorianos en España: Claves de un proceso migratorio en cuatro tiempos», Emiliano Gómez Ciriano, Andrés Tornos y Colectivo IOÉ, Ecuatorianos en España. Una aproximación sociológica, Madrid, Observatorio Permanente de la Inmigración, 2007: 15-99.

López, Nancy, «Race-Gender Experiences and Schooling: second-generation Dominican, West Indian and Haitian youth in New York City», Race Ethnicity and Education, 5/1 (Londres, 2002): 67-89.

Loui, Vivian, «Second-Generation Pessimism and Optimism: How Chinese and Dominicans Understand Education and Mobility Trough Ethnic and Transnational Orientations», International Migration Review 40/3 (2006) 537-572.

Ministerio de Trabajo e Inmigración, Anuario Estadístico de Emigración 2006. Madrid, Ministerio de Trabajo e Inmigración, Online, 8-10-08. http://extranjeros. mtin.es/es/InformacionEstadistica/Anuarios/Anuario2006.html.

Ministerio de Trabajo e Inmigración, Informes Trimestrales 1/2008. Extranjeros con certificado de registro o tarjeta de residencia en vigor y Extranjeros con autorización de estancia por estudios en vigor a 31 de marzo de 2008, Online, 13-10-08. http:/extranjeros.mtin.es/es/InformacionEstadistica/Anuarios/Anuario2007.html. 
Ministerio de Educación, Política Social y Deporte. Estadística de las Enseñanzas no universitarias (Centros, Personal, Alumnado matriculado y Graduados). Online, 20-10-08. http://www.mepsyd.es/mecd/jsp/plantilla.jsp?id=310\&area=estadisticas \&contenido=/estadisticas/educativas/eenu/avances/Curso07-08/avances.html

Suárez-Orozco, Marcelo y Suárez-Orozco, Carlota, La infancia de la inmigración. Madrid, Morata, 2003.

Truong, Anhhuy, «Perceived Discrimination, Linguistic Acculturation, and Psychological Well-Being Among Second Generation Immigrant Youths», Paper presented at the annual meeting of the American Sociological Association, Hilton San Francisco \& Renaissance Parc 55 Hotel, San Francisco, CA, Aug 14, 2004.

Online <.PDF>. 2008-10-14 http://www.allacademic.com/meta/p108311_index.html

\section{THE MIGRATORY FLOOD STARTS TO SETTLE}

The flows of immigrants in Spain have been continuous and increasingly numerous for the past fifteen years. A possible aftermath of such process may consist in the consolidation of minorities in our country. This paper intends to analyse some of the settling and consolidation processes of a population, the Ecuadoran population, who has been involved in a significant entry flow. It is basically focused on the adaptation strategies of the Ecuadoran immigrants' children in the familiar and educational domains.

KEY WORDS: Immigrants' children, Ecuadoran, minorities, styles of breeding, education. 